UDC 633.63.631.35

(C) 2016

V. Bulgakov, Academician of the National Academy of Sciences of Ukraine, Doctor of Technical Sciences, National University of Bioresources and Natural Resources of Ukraine

V. Adamchuk, Academician of the National Academy of Sciences of Ukraine, Doctor of Technical Sciences, National Science Center "Institute of Mechanization and Electrification of Agriculture"

Ye. Ignatyev

Tavriya State Agrotechnological University

\title{
THEORY OF CLEANING HEADS OF ROOT CROPS OF BEET BY ELASTIC CLEANING BLADE
}

Key words: sugar beet, haulm, cleaning blade, theory, equivalent scheme, force, stroke, differential equations, parameters.

\section{Formulation of the problem.}

The use of modern technology for the harvesting of sugar beet hinges suggests that after the main continuous cutting of the sugar beet hinges, usually rotary hiccircular machines, the roots of the root crops remain the remains of the hinges in the form of green and strong short petioles (stems) or dry and lingering individual leaves and stems, located in the row spacings of crops. This significantly and unacceptably contaminates the root crops of sugar beet during their subsequent excavation from the soil [1]. Therefore, for the purpose of the inadmissibility of the content of plant impurities in a heap of dug roots, the designs of the head cleaners on the root were created, of which the lobe cleaners were the most widespread, with driven horizontal shafts. By their constructive performance, such cleaners can be single or double-walled. Thus, the design of the most sophisticated dual-purpose cleaner contains successively located two drive shafts with elastic cleaning blades installed on them, which during the process move progressively along rows of crops at a fixed height parallel to the surface of the soil and are located at a certain angle to the rows of beet root crops and purified from 
the residues tops of head of root crops. On the first shaft, perpendicular to its axis of rotation, the console rigidly fixed flexible shovel blades from the rubberized belt. On the second purifying shaft are used cleaning blades of rubber, which are fixed hinged and located in the radial direction. The first drive shaft rotates against the direction of the forward movement of the cleaner, and the second, on the contrary - in the course of the movement of the cleaner. Flexible shovels of the first shaft, striking the heads of the root crops of sugar beets, shreds the remnants of the hoof from the heads of the root crops, and, due to the location of the shaft at a certain angle to the rows of root crops of sugar beet, the plant (and also shallow soil) impurities from the plantation area prepared for excavation of root crops from the soil to the removed area of beet field. Cleaning the shovels of the second shaft, rotating towards the shovels of the first shaft, finally cleans the heads of the root crops from the uncut bits.

As numerous tests have shown, this dual purifier has a number of significant disadvantages, among which the main ones are: increased energy intensity of the process, the cutting of beet root crops from the soil, which further leads to their losses, there is an intense wear of elastic cleaning blades, which greatly complicates the operation cleaner Not in all cases, these types of head cleaners root sugar beet root provide the required quality of cleaning. So, in case when on the spherical surface of the head of the root remain uncut short, green and strong remnants of the hinge, the elastic blades, unfortunately, are not in a position, by a single effect, to effectively remove them from the heads of the root crops. Also, quite often there are cases where not all spherical surface of the head of the root crop is equally qualitatively cleared from the remnants of the hinge, especially the part that lies behind the direction of the translational motion of the cleaner. Therefore, the technological process of cleaning root crops from the remains of the hinge at the root of an elastic cleaning blade installed on the drive horizontal shaft requires its further improvement, in particular, a more profound study and substantiation of optimal structural and kinematic parameters that ensure their high 
efficiency.

\section{Analysis of recent research and publications.}

A number of scientific works [2-6] and others are devoted to issues of cleaning (removing the remains of tops) from sugar beet root crop heads after its main cutting at the root of cutting machines of hoeing machines and head cleaners. The results of theoretical and experimental investigations of sugar beet root crop heads of various designs are presented: lobed [2-4], ring, sector, drum types, and also a purifier made in the form of a paraboloid [5, 7]. However, the greatest use in the world was found in the cleaners of heads of root crops of the blade type, due to the simplicity of their designs and the high quality of work in relatively favorable conditions (lack of a significant number of remnants of the hive - short, green and dry, dead, firmly located on the heads of root crops of sugar beet). Different variants of theoretical investigations of the interaction of the treatment elements with the root of sugar beet root, the parameters of the hinge and the technological schemes of the hoeing machines are presented in numerical published scientific papers $[8,9]$. However, in most of the above mentioned scientific works, in the theoretical substantiation of their constructive and kinematic parameters, the combining effort created by the purifiers, the dimensional characteristics of the roots, the direction and the module of the working speed of the translational motion are not accurately and fully taken into account. It is also not enough, in some works, the weight of elastic cleaning blades, etc., is substantiated. Despite the fact that the technological processes of harvesting sugar and fodder beet are largely similar, however, the harvesting of the hinge and its remains from the root crops of fodder beet at the root is practically not investigated, in some works partly substantiated and sometimes experimentally determined their parameters [10, 11] . It should also be emphasized that the information given in the scientific literature relates to theoretical and experimental studies of only specific types and designs of head cleaners of root crops from the remnants of the hinge with all assumptions and simplifications adopted by the authors, and therefore their application for 
further research into new types of purifiers is rather complicated. The main provisions of the refined theory of the interaction of a flexible cleaving blade with a head of root crop, fixed in the soil are given in [3]. However, the results of numerical calculations that show the influence of the kinematic and constructive parameters of a flexible cleaving blade on the effectiveness of such interaction, that is, on the quality of cleaning the roots of sugar beet root roots from the remnants of the hinge, are not presented here.

Thus, so far, the literature did not find a reflection of the research that contained the most general and complete, and therefore the universal theories that could be used for numerical simulation and which could serve as the basis for further calculations and the design of any type of root beet head cleaners on The roots of the elastic cleaning blade, mounted on the drive horizontal shaft.

\section{The aim of the study.}

Develop the most general theory of the interaction of an elastic cleansing blade with a head root of sugar beet at the root, in the process of its purification from the remains of the hinge, when the blade is installed on the drive horizontal shaft and give the results of numerical simulation of its kinematic and structural parameters.

\section{Research methods.}

An analytical study was carried out using simulation methods, higher mathematics and theoretical mechanics, in particular the theorems on the change of the kinetic energy of a mechanical system, the theorem on the change of the moment of the amount of motion, the theory of impact, assembly and solution in the closed form of differential equations of motion. Numerical calculations of the received new analytical studies conducted using the methods of drawing up programs and calculations on the PC.

\section{Results of the research and their discussion.}

For the theoretical substantiation of the optimal structural and kinematic parameters of root crop head cleaners from the residual joints with horizontal drive 
shafts, we will construct the most general mathematical model of the interaction of the elastic cleansing blade of the cleaner with the head of the root crop of sugar beets fixed in the soil.

For this purpose, in the beginning, we will form the equivalent scheme of the simple blade cleanser of the heads of sugar beet root crops, that is, consider only the process of interaction of a unit, elastic cleansing blade with head of root crop of beet located (fixed) in the soil, whose head protrudes to a certain height above the surface of the soil It contains, on its own, non-cut remains of the hinges (petioles) (Fig. 1).

When constructing the equivalent scheme of the interaction of an elastic cleaning blade installed on a drive horizontal shaft with a root head of sugar beet, that is, actually the simplest lober cleaner, we have adopted the following assumptions:

1) The elastic cleaning blade $A M$ is homogeneous throughout its length;

2) at the contact of the cleaning blade with the sugar beet roots, the interaction of forces occurs only at the point $M$ of contact with the root of the root;

3) the permissible force $\bar{P}$ of impact of the elastic cleansing blade on the head of the root crop does not exceed the permissible force on knocking (twisting) of the root crop of sugar beet from the soil, that is $P \leq[P]$, where $[P]$ - the permissible force for cutting root crop of beet from the soil;

4) the air resistance during rotation of the cleaning blade is not taken into account.

The mentioned process of interaction of the elastic cleansing blade and head of the root sugar of sugar beet will be considered in a longitudinal-vertical plane. In this case, the axis of the horizontal drive shaft (point $O$ on an equivalent scheme), the cleaner is perpendicular to the longitudinal vertical plane; the $A M$ shaft is suspended horizontally on the $A$ axis, which stands from the center of the drive shaft by the value of the radius of rotation $r$. The end of the blade describes a circle with a radius $\rho$ when rotated with a constant angular velocity $\omega$ in a 
longitudinal vertical plane. The axis of the drive horizontal shaft (point $O$ ) moves above the level of the soil surface at a constant height $\mathrm{H}$ of the installation. The rotation axis $O$ also moves progressively with constant speed $\bar{V}_{o}$. We connect from the axis of the drive horizontal shaft (point $O$ ) a rectangular Cartesian coordinate system $x O z$ in which the horizontal axis $O x$ coincides with the direction of the forward movement of the cleaner, and the axis $\mathrm{Oz}$ is directed upwards. In this case, the length of the elastic cleaning blade is $2 l$. Point $M$ is the point of contact of the blade on the head of the root crop.

The initial rate of impact of the elastic cleaning blade on the root of the root crop will consist of its relative velocity $\bar{V}_{r}$ at a rotary movement around the point $O$ and the speed at forward translational point $O$. There may be two possible variants. The first one, when the direction of rotation of the cleaning blade is carried out during the movement of the cleaner (Fig. 1), then the vectors of the relative speed $\bar{V}_{r}$ of the blade and the speed of the folding $\bar{V}_{o}$ are $\bar{V}=\bar{V}_{o}+\bar{V}_{r}$. The second option, when the direction of rotation of the blade is carried out against the course of the movement of the head cleaners root, then, in determining the speed of impact, the indicated vectors are subtracted $\bar{V}=\bar{V}_{r}-\bar{V}_{o}$. 


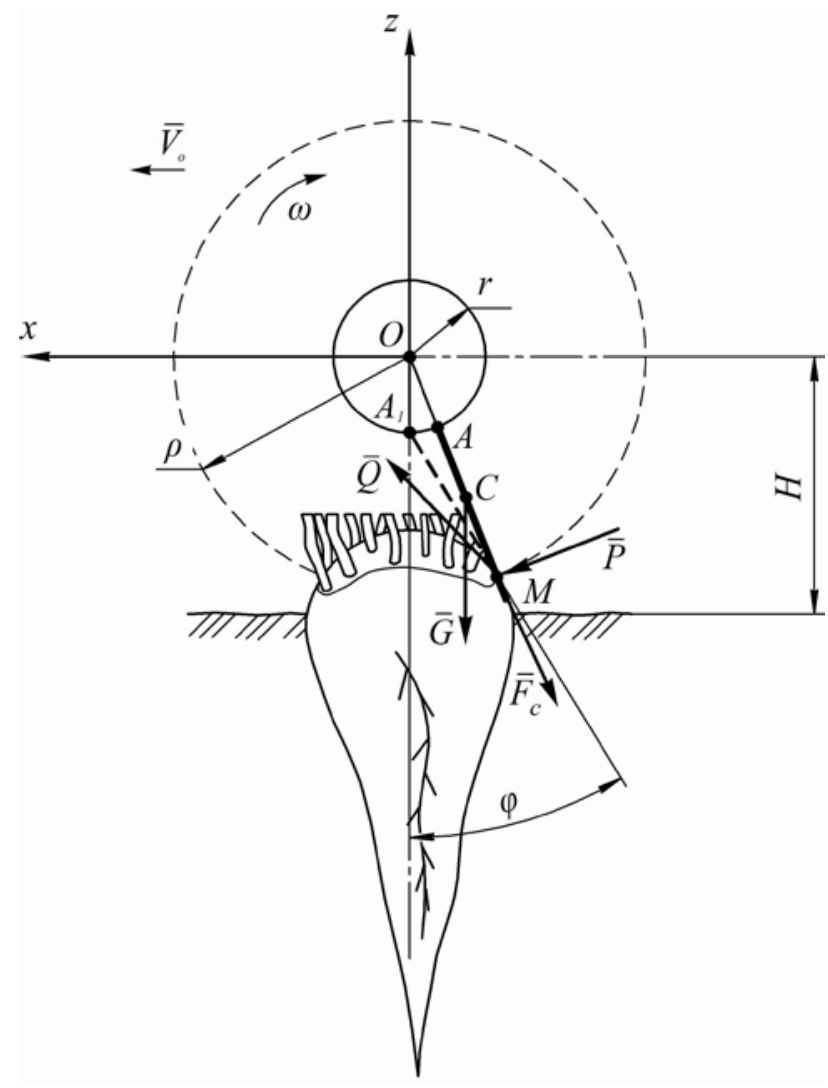

Fig. 1 - Equivalent scheme of interaction of the elastic cleaning blade installed on the drive horizontal shaft with head of sugar beet root

We will show on the equivalent scheme (Fig. 1) the forces acting on the interaction of an elastic cleansing blade with a root head of sugar beet at its cleansing on the root. We assume that at the point $M$ the force is applied $\bar{P}$ - the force of impact of the blade directed perpendicular to the $A M$ shovel itself at the very beginning of its interaction with the head of the root beet root. During the free rotation of the $A M$ blade around the axis (point $O$ ) with an angular velocity $\omega$ there is a centripetal force $\bar{F}_{c}$ equal to $F_{c}=m \rho \omega^{2}$ and directed along the $A M$ blade, which places the blade on one straight line with radius $r$ and hinge $A$. When the blade blows on the beet root head, in the phase of the contact, the force $\bar{G}$ of the blade weight itself will be equal to the blade itself: $G=m g$, which will be concentrated in the center of the masses of the cleaning blade (point $C$ ) at a distance $r+l$ from the axis of rotation and directed parallel to the vertical axis $\mathrm{Oz}$. 
For a further detailed analytical study, the technological process of cleaning beet root roots from the remnants of the gypsum is divided into two phases:

1) phase of the meeting of the blade blade with the root of the root beet;

2) the working phase of cleaning the head of the root of beet from the hive.

As the elastic cleaning blade $A M$ moves progressively along with its axis of rotation (point $O$ ) with a translatory velocity $\bar{V}_{o}$ of motion and simultaneously rotates around the same axis with an angular velocity $\omega$, then its kinetic energy $T$ will be equal to:

$$
T=\frac{m V_{o}^{2}}{2}+\frac{I_{o} \omega^{2}}{2}
$$

where $I_{o}$ - moment of inertia of the blade relative to the axis of rotation (point $O$ ).

The moment of inertia $I_{o}$ of the blade, according to Steiner's theorem, will be equal to:

$$
I_{o}=I_{c}+m(r+l)^{2},
$$

where $I_{c}$ - moment of inertia of the blade $A M$ relative to the axis of rotation passing through the center of the blade's mass (point $C$ ), parallel to the axis of rotation of the blade; $r+l-$ the distance from the center of the blade's mass to its axis of rotation.

Substituting expression (2) into (1), we obtain the value of the kinetic energy of the cleansing elastic blade before the start of its shock contact with the head of the root sugar of sugar beet:

$$
T=\frac{m V_{o}^{2}}{2}+\left[I_{c}+m(r+l)^{2}\right] \frac{\omega^{2}}{2} .
$$

However, at the moment of the start of the shock contact, point A, that is, the hinge of the suspension of the blade $A M$ to the drive shaft, continues to rotate around the axis of rotation of the drive shaft at an angular velocity $\omega$, and the $M$ point of contact of the end of the blade with the head of the root begins to slow down its rotation as a result of the same contact. Therefore, the cleaning blade begins to return to the opposite side of the point $A$, simultaneously sliding the 
lower end over the surface of the head of the root, thus, the process of combining the remnants of tops from the head of the root of sugar beet.

It is obvious that before the impact contact with the root head of the beet blade $A M$ is in one straight line with the radius $r$ of the drive shaft, connecting the point $O$ axis of the shaft with the point $A$ of the blade hinge, for an arbitrary time t returning to the angle $\omega t$, and after the contact With the head of the root crop, she begins to return to the corner $\omega t-\varphi$, where $\varphi$ - the angle of deviation of the blade from the position that would take this blade at free rotation around the point $O$. Therefore, in this case, the blade $A M$ will have an angular velocity equal to $\omega-\not \&$, and its kinetic energy $T$ in this case will be determined by such expression:

$$
T=\frac{m V_{o}^{2}}{2}+\left[I_{c}+m(r+l)^{2}\right] \frac{(\omega-\phi)^{2}}{2} .
$$

Comparing Expressions (3) and (4), we see that the part of the kinetic energy due to the dependence (3) turns into the energy of the impact and into the useful work of combining the remnants of the hinges from the head of the root sugar of sugar beet.

It is known that the time derivative from the kinetic energy of the material system is equal to the sum of the capacities of all external and internal forces applied to the system [12]. In particular, for absolutely solids, the sum of works, and hence the capacities of all internal forces, is zero.

Therefore, in this case, conditionally considering the lateral part of the head of the root of a completely solid body, we obtain the following equation of the balance of the power of the active forces acting on the root of the beet at the shock contact and spent with the kinetic energy:

$$
\frac{d T}{d t}=N
$$

where $N$ - total force of active forces acting on the sugar beet root of a time $t$.

It should be noted that the application of the theorem on the change in the kinetic energy of a mechanical system should not take into account the reactions of frictionless joints, since their work will be zero. 
Since the contact area of the blade with the head of the root is quite small compared with the length of the trajectory (circle of radius equal $r+2 l$ ), passing the lower end of the blade for one revolution around the axis of rotation, we can assume that in this section the end of the blade $A M$ moves progressively at a speed equal to $\bar{V}=\bar{V}_{o}+\bar{V}_{r}$. Moreover, due to the fact that the contact is carried out in the lower part of the circle (near the surface of the soil), in the first approximation we can assume that velocity vectors $\bar{V}_{o}$ and $\bar{V}_{r}$ are parallel, and therefore the geometric amount of these vectors can be replaced by algebraic, that is, $V=V_{o}+V_{r}$.

It is obvious that the relative velocity $\bar{V}_{r}$ of the end of the blade $A M$ before the start of the impact contact is equal to the magnitude $V_{r}=\omega(r+2 l)$, and during the contact period it is equal $V_{r}=(\omega-\phi)(r+2 l)$, where $r+2 l$ is the distance from the $M$ point of contact to the $O$ axis of rotation of the drive shaft. Thus, in the first case $V=V_{o}+\omega(r+2 l)$ :, and in the second: $V=V_{o}+(\omega-\phi)(r+2 l)$.

In addition, it is obvious that the weight $\bar{G}$ of the $A M$ shovel is too small in comparison with the impact force $\bar{P}$ at the beginning of the contact of the blade with the head of the root crop and is small compared with the force $\bar{Q}$ of combining the remnants of the hinges from the head of the root in the period of contact, and therefore it can be neglected in the balance of power and energy

Thus, at the beginning of the contact we have equality: $N=P V$, or $N=P\left[V_{o}+\omega(r+2 l)\right]$, and in the period of the contact - equality: $N=P\left[V_{o}+(\omega-\phi)(r+2 l)\right]$.

Then equation (5) of the balance of the power of the impact force $P$ and the losses of kinetic energy during the impact will have the following form:

$$
\frac{d T}{d t}=P\left[V_{o}+\omega(r+2 l)\right]
$$

Differentiating the expression (4) in time $t$, we obtain: 


$$
\frac{d T}{d t}=\left[I_{c}+m(r+l)^{2}\right](\omega-\phi)
$$

Equating the right-hand sides of expressions (6) and (7), we will have:

$$
\left[I_{c}+m(r+l)^{2}\right](\omega-\infty)=P\left[V_{o}+\omega(r+2 l)\right] \text {. }
$$

Let's continue $[P]$ - the permissible force of knocking out the root sugar of sugar beet from the ground with the shock interaction of the elastic cleansing blade with the head of the root crop. Replacing the impact force $P$ in the expression (10) with the permissible shock force, we obtain the differential equation of rotation of the elastic cleansing blade around point $\mathrm{A}$, provided that the root beet is not removed from the soil at the beginning of the shock contact, that is, during a very small time interval t, more precisely, the time of the shock contact. The next, postimpact angular displacement of the blade will be described by the following differential equation

Let's continue - the permissible force of knocking out the root sugar of sugar beet from the ground with the shock interaction of the elastic cleansing blade with the head of the root crop. Replacing the expression (10) with the percussion P on the permissible shock force $[P]$, we get the differential equation of rotation of the elastic cleansing blade around point $A$, provided that the root beet is not removed from the soil at the beginning of the shock contact, that is, during a very small time interval $t$, more precisely, the time of the shock contact. The next, post-impact angular displacement of the blade will be described by the following differential equation:

$$
\left[I_{c}+m(r+l)^{2}\right](\omega-\phi) Q\left[V_{o}+(\omega-\phi)(r+2 l)\right],
$$

where $Q$ - the power of combing the remnants of tops from the root of sugar beet root.

The differential equation (8) can be replaced by the difference equation, using the theorem on the change of the moment of the amount of motion of a mechanical system under shock interaction: 


$$
I_{A} \omega_{1}-I_{A} \omega_{o}=M_{A}(S),
$$

where $I_{A}$ - moment of inertia of the blade relative to the point $A ; \omega_{o}$ - angular velocity of the blade relative to the point $A$ to impact; $\omega_{1}$ - angular velocity of the blade relative to the point $A$ after impact; $M_{A}(S)$ - moment of momentum of the impact force relative to the point $A$.

In this case, the impact pulse $S$ will be equal to:

$$
S=\int_{0}^{\tau} P d t
$$

and the moment of the given shock impulse will have this value:

$$
M_{A}(S)=2 l \int_{0}^{\tau} P d t
$$

where $P$ - impsct force; $2 l$ - blade length; $\tau$-impact duration.

As the elastic cleaning blade did not return to the point of contact until the impact contact $A$, because $\omega_{o}=0$. Then, from the expressions (10) and (12) with $\omega_{o}=0$, we find the angular velocity $\omega_{1}$ of the blade in the angular displacement relative to the point $A$ after the impact:

$$
\omega_{1}=\frac{2 l \int_{0}^{\tau} P d t}{I_{A}} .
$$

If we replace the force $P$ by the force $[P]$, and take into account that $[P]=$ const , then we get:

$$
\omega_{1}=\frac{2[P] l \tau}{I_{A}},
$$

where $I_{A}=I_{c}+m l^{2}$.

Thus, the angular velocity $\omega_{1}$ of the elastic cleansing blade relative to the point $A$ after the impact is determined, provided that the root sugar of the sugar beet is not sliced from the ground. Therefore, the angular velocity of the cleaning blade in the rotary motion relative to the point $O$ after impact will be equal $\omega-\omega_{1}$. 
Assume in equation (9), in the first approximation that $\& \&=\omega_{1}$. This assumption can be made due to the short-term contact of the cleaning blade with the root of the root beet. Then equation (9) is much simpler and it is possible to determine the angular acceleration of the blade which will be equal to:

$$
=\frac{Q\left[V_{o}+\left(\omega-\omega_{1}\right)(r+2 l)\right]}{\left[I_{c}+m(r+l)^{2}\right]\left(\omega-\omega_{1}\right)} .
$$

After the first and second integration of expression (15), taking into account that the arbitrary constants $C_{1}$ and $C_{2}$ obtained during the integration and are determined from the initial conditions, that is, at $t=0: \& \&=\omega_{1}, \varphi=0$, and therefore $C_{1}=\omega_{1}, C_{2}=0$, receive the law of the angular post-impact movement of the elastic cleansing blade on the head of the root of sugar beet, in which the combing is carried out Remnants of a gimlet from its head

$$
\varphi=\frac{Q\left[V_{o}+\left(\omega-\omega_{1}\right)(r+2 l)\right] t^{2}}{2\left[I_{c}+m(r+l)^{2}\right]\left(\omega-\omega_{1}\right)}+\omega_{1} t .
$$

The force $Q$ of combining the remnants of a hive from the head of the root beet analytically will be determined in this way. As noted in $[8,11]$, the cuttings of the hinges in the cross-section, in the general case, are close to the triangular shape, which has at the base of the depression of the same triangular shape (Fig. 2).

Indicated in fig. 2 sizes are used later to calculate the cross-sectional area of the petioles of sugar beet hooks that need to be cleaned (removed) by an elastic cleaning blade

We will assume that the process of combing the remnants of the hinge occurs directly on the head of the root crop at the point of exit of the stalk from the head, that is, the attachment of the stalk, due to the deformation of the direct shift of the petiole itself. It is obvious that the process of combing will be possible under the condition when $\frac{Q}{n F} \geq[\tau]$, where $Q$ is the combing power; $[\tau]$ - the permissible tangential stresses of the shift for the stem of the hinge; $F$ - cross-sectional area of 
one petiole; $n$ is the number of petioles that are simultaneously coupled from the spherical surface of the head of the root sugar of sugar beet

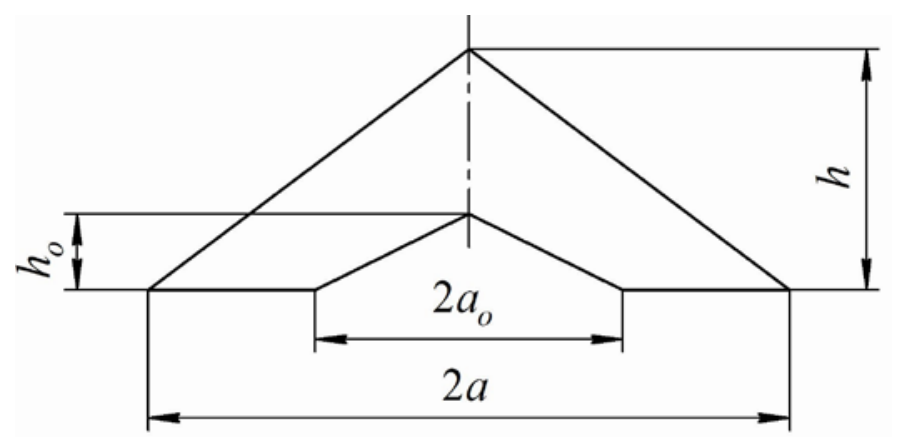

Fig. 2 - Scheme of the cross section of the petiole of sugar beet hinges

Determine further, proceeding from this, necessary to combine the remnants of the jug from the head of sugar beet roots, the force of combing $Q$. If we consider that, then $F=a h-a_{o} h_{o}$ it will equal:

$$
Q \geq\left(a h-a_{o} h_{o}\right) n[\tau] .
$$

In the first approximation, we assume that a row consisting of five petioles is joined simultaneously. According to $[8,11]$, the cake of a honeycomb of sugar beet has the following dimensions: $a=5 \mathrm{~mm} ; a_{o}=2 \mathrm{~mm} ; h=5 \mathrm{~mm} ; h_{o}=2 \mathrm{~mm}$. The average value of the permissible tangent stress $[\tau]$ for the material of the trunk of the tie in accordance with [11] is: $[\tau]=1.14 \cdot 106 \mathrm{~Pa}$

Substituting the concrete values of the above quantities in expression (17), we obtain that the value of the combing force will be equal: $Q=120 \mathrm{~N}$.

Asking for the time of contact $t_{1}$ of an elastic cleaning blade with a head of sugar beet root is the possibility to find the angle of rotation $\varphi_{1}$ of the blade around point $A$ during the contact, from which it is finally possible to determine the angular velocity $\omega$ of the rotation of the cleaning blade around the point $O$, taking into account the condition of not knocking out the root sugar of the sugar beet from the ground in the process of combining the residues of the hinges from the head of the root: 


$$
\omega=\frac{Q V_{o} t_{1}^{2}}{2\left[I_{c}+m(r+l)^{2}\right]\left(\varphi_{1}-\omega_{1} t_{1}\right)-Q(r+2 l) t_{1}{ }^{2}}+\omega_{1} .
$$

In the same way, you can calculate other parameters of the elastic cleaning blade, for example, the mass of the $m$ blade or its length of $2 l$.

The analytical expressions obtained include the moment of inertia $I_{c}$ of the blade relative to the axis passing through the center of the blade's mass (point $C$ ) parallel to the axis of rotation of the drive shaft (point $O$ ), which depends on the shape of the cross-section of the elastic blade itself.

For an elastic cleaning blade, which has the shape of a rectangular parallelepiped with the sides $2 a \times 2 b \times 2 l$, the indicated moment of inertia $I_{c}$ will be equal to $I_{c}=\frac{m}{3}\left(a^{2}+l^{2}\right):$, where $2 a$ is the width of the blade; $2 l$ is the length of the blade, if the cleaning blade is in the form of a straight round cylinder $I_{c}$, then its moment of inertia will be equal to $I_{c}=\frac{m}{4}\left(\frac{4 l^{2}}{3}+r_{1}^{2}\right)$ :, where $r_{1}$ - the radius of the cross section of the blade; $2 l$ is the length of the blade [12].

Using the above mentioned values of the moments of inertia $I_{c}$ and substituting them in expression (20), it is possible to determine the dependence of $\omega$ on other kinematic and constructive parameters of the process of cleaning the sugar beet root crops heads by an elastic cleaning blade installed on the drive horizontal shaft

Thus, as a result of the theoretical research, the analytical expressions that give the possibility to directly determine the structural and kinematic parameters of the elastic cleansing blade, which will ensure the effective combining of the residuals of tops from the root crops of sugar beet, provided that the root crops are not cut from the ground, are obtained from the ground the bulk of the hook.

After compiling the program on a PC in the Mathcad environment, numerical calculations of the kinematic parameters of the technological process of cleaning the sugar beet root of the head of root were carried out, depending on the 
structural parameters of the blade cleaner itself, the physical-mechanical properties of the hinge and the conditions for not knocking out the root of the root itself.

The data of these calculations are presented in Fig. 3-5.

From the graphs we see that the angular velocity $\omega$ of the rotary motion of an elastic cleaning blade has a dependence on its length $2 l$ close to the exponential form. In this case, the mass of the blade $m$ almost does not affect this dependence. At the same time, as we see from the graphs, to improve the impact of the length of $2 l$ blade on the value of the angular velocity $\omega$ of the rotary motion of the elastic cleaning blade, it is necessary to choose the length of the blade close to $0,3 \ldots 0,4$ $\mathrm{m}$, which will provide the minimum values of the angular velocity $\omega$, at which there is a qualitative cleansing of the heads of root crops from the remains of tops and there is no knocking out of the bodies of root crops from the soil

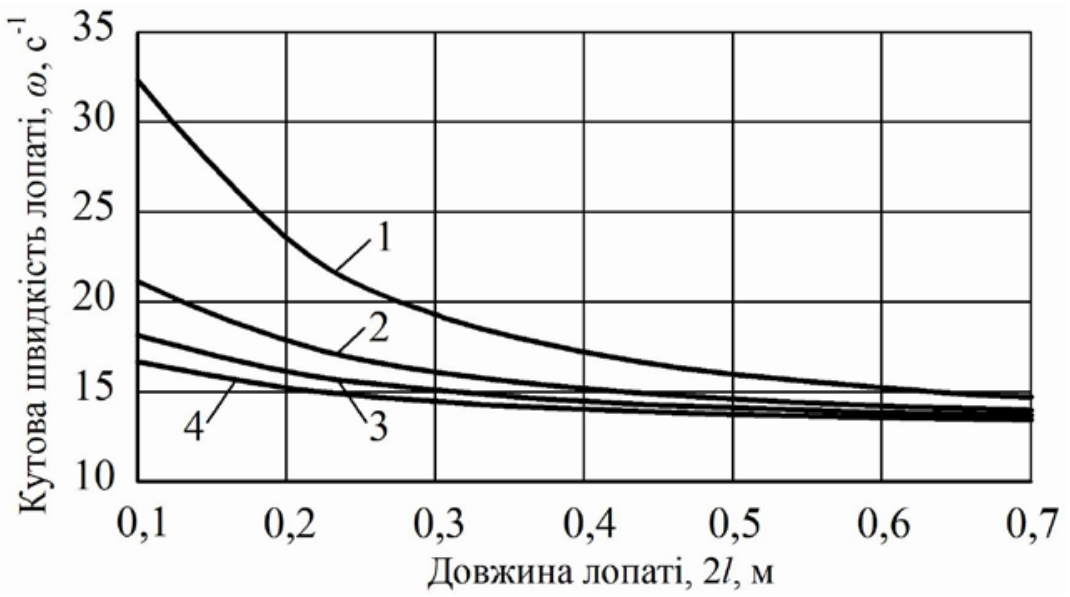

Fig. 3 - The dependence of the angular velocity $\omega$ on the length of $2 l$ blades with different values of its mass:

$$
1-m=0,25 \mathrm{~kg} ; 2-m=0,40 \mathrm{~kg} ; 3-m=0,55 \mathrm{~kg} ; 4-m=0,70 \mathrm{~kg}
$$

With regard to the dependence of the angular velocity $\omega$ on the radius of the suspension of the blade, the increase in the value of the specified design parameter will also provide the minimum values of the angular velocity $\omega$ in the case of application of a predetermined length of $2 l$ elastic cleaning blade. Thus, the drum of the head cleaners of root crops from the remnants of the hinge at the root should have a radius that will be not less than $0.30 \mathrm{~m}$ for rational values of the length of the blade $2 l$.. 


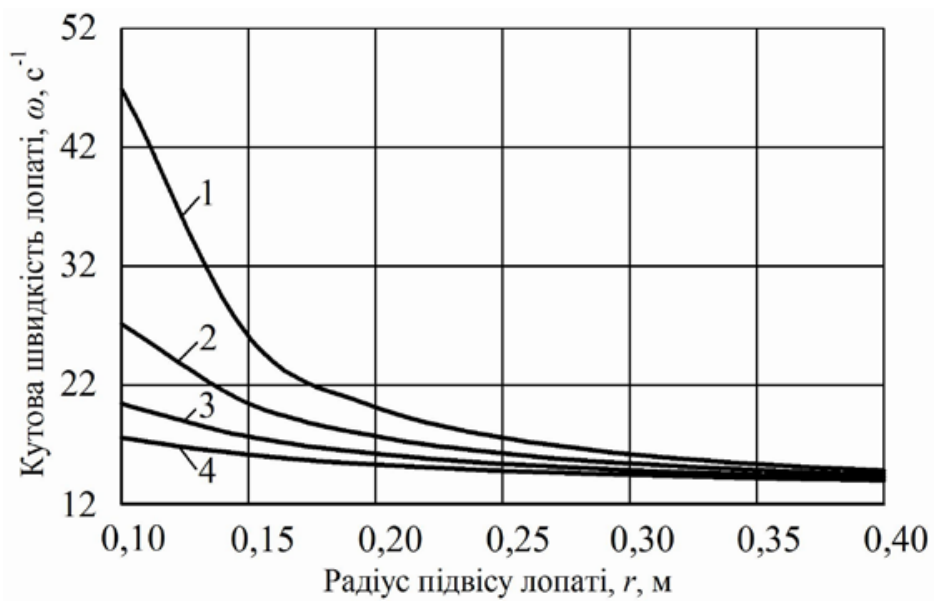

Fig. 4 - The dependence of the angular velocity $\omega$ on the radius $r$ of the suspension

blades at different values of the length of the blade $2 l$ :

$1-2 l=0,1 \mathrm{~m} ; 2-2 l=0,2 \mathrm{~m} ; 3-2 l=0,3 \mathrm{~m} ; 4-2 l=0,4 \mathrm{~m}$

The dependence of the angular velocity $\omega$ on the velocity $V_{o}$ of the translational motion of the elastic cleansing blade is linear. At the same time there is an inject on the specified dependence of the length $2 l$ of the cleaning blade itself. However, as we see from the graphs, the most expedient is the translational velocity $V_{o}$, which does not exceed $2.5 \ldots 3.0 \mathrm{~m} \cdot \mathrm{s}^{-1}$. In this case, the length of $2 l$ elastic cleaning blade should also be not less than $0,4 \mathrm{~m}$

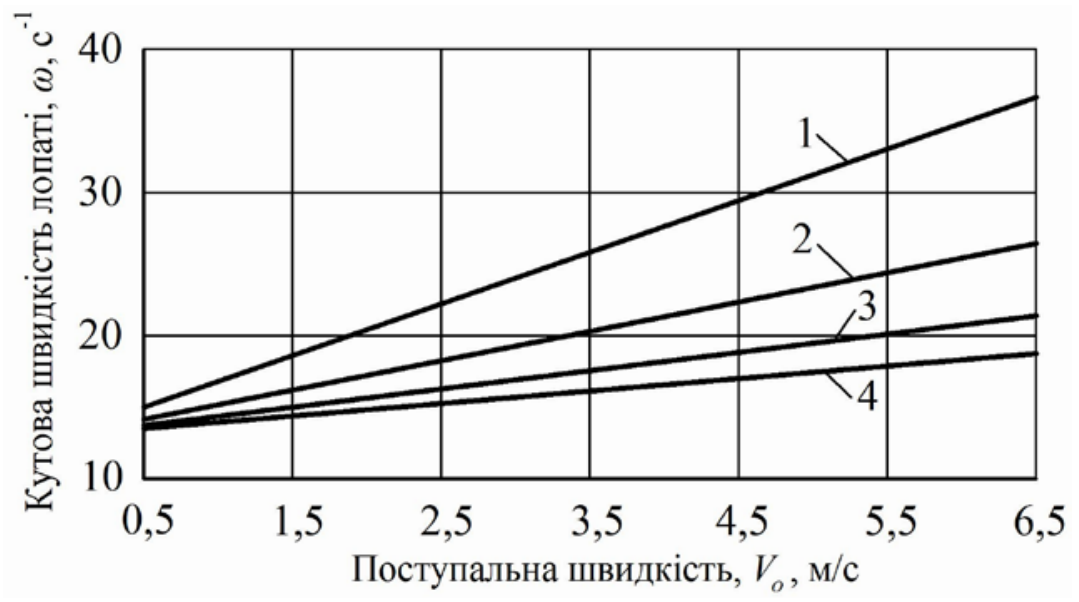

Fig. 5 - The dependence of the angular velocity $\omega$ on the velocity $V_{o}$ of the translational motion at different values of the length of the blade $2 l$ :

$$
1-2 l=0,1 \mathrm{~m} ; 2-2 l=0,2 \mathrm{~m} ; 3-2 l=0,3 \mathrm{~m} ; 4-2 l=0,4 \mathrm{~m}
$$

Thus, on the basis of numerical simulation on the PC, it has been established 
that the most rational kinematic and constructive parameters of the head cleaners of root crops of sugar beet at the root, which provide high quality indicators of cleaning the heads from the remains of the hinge and not knocking out the bodies of root crops from the soil are: speed $V_{o}$ of translational motion is not less than $2,5 \ldots 3,0 \mathrm{~m} \cdot \mathrm{s}^{-1}$, legth $2 l$ of elastic cleaning blade should be at least as high as $0,3 \ldots 0,4 \mathrm{~m}$, and the radius $r$ of the drum (that is, the radius of the suspension of the cleaning blade) is also not less than $0,3 \mathrm{~m}$.

\section{Conclusions:}

1. The cleansing of sugar beet root crops from the remains of the hiccule at the root is an up-to-date and difficult task for the field of mechanization of beetroot, which has not yet found an effective, precise and final solution. Therefore, theoretical and experimental researches that allow to determine the optimal structural and kinematic parameters of the working bodies of the heads of root crops from the remnants of the hinges allow not only to improve the given technological process, but also give an opportunity to create new, more effective samples of cleaners.

2. A new theory of the interaction of a flexible cleaning blade installed on the drive horizontal shaft and the head of the root beet fixed in the soil is based on the application of the theorems on the change of the kinetic energy of the mechanical system and on the change in the amount of motion, as well as the impact of the theory, gave a high degree of opportunity the accuracy of the simulation of the specified purification process, taking into account the creation of the required combing power and the conditions for not cutting the root beet from the soil.

3. The final solution of the differential equation of motion of a flexible cleavage blade along the root of the root beet gave an opportunity to obtain new analytical expressions for determining the angle of rotation and angular velocity of the rotary motion of the blade blade installed on the drive horizontal shaft from the conditions of efficient combining the remnants of the hinge and not the destruction 
of the bonds with a root of beet root, with different meanings of its geometric sizes and shapes: a rectangular parallelepiped and a straight circular cylinder. A new analytical expression has also been obtained to determine the force of combining the petioles of the jug with the spherical surface of the head of the sugar beet root.

4. The results of numerical simulation on the PC showed that the rational kinematic and constructive parameters of the head cleaners of sugar beet root roots at the root, which provide high quality indicators of cleaning the heads from the remnants of the hinge and not knocking out the bodies of root crops from the soil are: speed $V_{o}$ of translational motion is not less than $2,5 \ldots 3,0 \mathrm{~m} \cdot \mathrm{s}^{-1}$, legth $2 l$ of elastic cleaning blade should be at least as high as $0,3 \ldots 0,4 \mathrm{~m}$, and the radius $r$ of the drum (that is, the radius of the suspension of the cleaning blade) is also not less than $0,3 \mathrm{~m}$.

\section{Bibliography}

1. Lammers S. Defoliation of sugar beets - assessment of quality and gain in delivered beet mass/S. Lammers, P. Olaf, R. Olaf//Landtechnik. - 2010. - №6. P. 464-467.

2. Pogorely L.V. Beet harvesting machines/L.V. Pogorely, N.V.Tatyanko, V.V. Brey and others - Kyiv: Tekhnika, 1983. - 168 p.

3. Bulgakov V. Theoretical investigations in cleaning sugar beet heads from remnants of leaves by cleaning blade/V. Bulgakov, S. Ivanovs, I. Golovach, Z. Ruzhylo. - 15 -th International scientific conference "Engineering for rural development”, Proceedings, Vol. 15. May 25-27, 2016. Jelgava. - P. 1090-1097.

4. Linnik A. Determination of dynamic parameters of rigid cleaner in the interaction with root crops/A. Linnik//Bulletin of TNTU. - 2014. - Volume 73. - № 1. - P.165-171.

5. Smith L. The effect of defoliator flail configuration, speed and crown removal on sugarbeet yield, quality and profitability/L. Smith//Sugarbeet Research and Extension Reports. - 1991. - Vol. 22. - P. 222-227.

6. Lilleboe D. Optimizing defoliator \& harvester 
performance/D. Lilleboe//The sugarbeet grower. - July 2014. - Vol. 53., №6. - P. 6-13.

7. Martynenko V. Ya. Justification of the constructive parameters of root head cleaners/V. Ya. Martynenko//Materials of the international scientific and practical conference: "Problems and prospects for the creation of beet harvesting equipment". - Vinnitsa, 1996. - C. 41-44.

8. Adamchuk V. Laboratory and field equipment workingout and the results of experimental studies of pre-harvesting sugar beet field conditions/V. Adamchuk, V. Bulgakov, M. Korenko et al.//Mechanization in agriculture. - Sofia, 2016. - Issue 1. - P. 3-5.

9. Ihnatiev Ye. I. Development of new constructive and technology scheme of sugar beet tops harvesting with use of arable and row-crop tractor/Ye.I. Ihnatiev//News of agrarian sciences. - 2016. - № 8. - P. 67-71.

10. Gurchenko A.P. Mechanization of harvesting sugar beets/AP Gurchenko, Ya. V. Savchenko//Engineering in agriculture. - Moscow: Urojai, 1986. - № 9. - P. 15-17.

11. Helemendic M. M. Directions and methods of working out of working bodies of agricultural machines. Monograph/M. M. Helemendik. - Kyiv: Agrarian Science, 2001. - 280 p.

12. Buthenin N.V. The course of theoretical mechanics/N.V. Buthenin, Ya.L. Lunts, D.R. Merkin. - Volume 2. - Moscow: Science, 1985. - 496 p. 